\title{
Study on Tag, Trust and Probability Matrix Factorization Based Social Network Recommendation
}

\author{
Zhigang Liu ${ }^{1}$ and Haidong Zhong ${ }^{2}$ \\ ${ }^{1}$ Control Technology Institute, Wuxi Institute of Technology, Wuxi, Jiangsu, 214121, P.R. China \\ [e-mail: liuzhigang510@126.com] \\ ${ }^{2}$ Logistics and e-commerce school, Zhejiang Wanli University, \\ Ningbo, Zhejiang, 315100, P. R. China \\ [e-mail: zhonghaidong@zwu.edu.cn] \\ *Corresponding author: Haidong Zhong
}

Received June 21, 2017; revised September 14, 2017; accepted November 8, 2017;

published May 31, 2018

\begin{abstract}
In recent years, social network related applications such as WeChat, Facebook, Twitter and so on, have attracted hundreds of millions of people to share their experience, plan or organize, and attend social events with friends. In these operations, plenty of valuable information is accumulated, which makes an innovative approach to explore users' preference and overcome challenges in traditional recommender systems. Based on the study of the existing social network recommendation methods, we find there is an abundant information that can be incorporated into probability matrix factorization (PMF) model to handle challenges such as data sparsity in many recommender systems. Therefore, the research put forward a unified social network recommendation framework that combine tags, trust between users, ratings with PMF. The uniformed method is based on three existing recommendation models (SoRecUser, SoRecItem and SoRec), and the complexity analysis indicates that our approach has good effectiveness and can be applied to large-scale datasets. Furthermore, experimental results on publicly available Last.fm dataset show that our method outperforms the existing state-of-art social network recommendation approaches, measured by MAE and MRSE in different data sparse conditions.
\end{abstract}

We express our thanks to Dr. Richard Berke who checked our manuscript. 
Keywords: Social network recommendation, Social tag, Social trust, Probability matrix factorization

\section{Introduction}

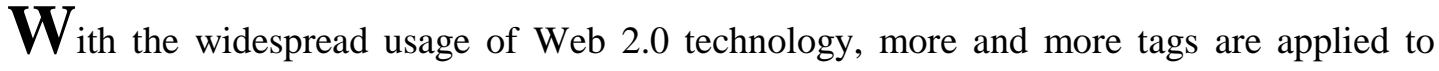
annotate resources such as a web page, a blog post or an image, with a set of keywords that the participants can define at will. These keywords play an important role in navigating and information retrieving [1]. Meanwhile tag based recommendation attracts much attention of researchers all over the world to improve the efficiency or solve challenges in traditional recommender technologies such as collaborative filtering, content-based filtering and hybrid recommendation. Wu L. et al. incorporated user-tag and item-tag interaction matrixes and proposed a two-stage recommendation framework, namely, NHPMF (Neighborhood-aware Probabilistic Matrix Factorization) model to improve recommendation accuracy [2]. Zhou T. C. et al. put forward a uniformed recommender framework (TagRec), based on probabilistic matrix factorization by utilizing both users' tags information and rating knowledge [3]. In [4], SoRecUser model was put forward to associate rating information with tag information by sharing user latent feature vector, and SoRecItem method was proposed to combine rating information with item information by sharing item latent feature vector.

In recent years, the enormous popularity of social network applications like Facebook, WeChat and Twitter, have provided a new way to communicate, collaborate and share information. Nowadays, social networks related applications have changed our everyday experience. Moreore and more people are incline to spread message by means of multitudinous social media platforms [5, 6]. This sprouts a great number of new application domains, and many traditional applications extend their social network compatibility. Social network based recommendation (SNR) is undoubtedly belongs to the latter case, which makes recommendations for a target user based on ratings of the individuals that have direct or indirect social relationships with the user [7]. Many existing recommendation methods can be extended by incorporating the interaction among users into them to meet the feature of social networks. However, recent studies show that social networks are highly dynamic, growing and changing very quickly [8]. Therefore, SNR still suffers data sparsity and other problems caused by the particularity of social networks.

To improve accuracy and effectiveness in SNR, many scholars put a lot of effort into social trust and social tag based recommendation. For example, Jamali M. et al. proposed a matrix factorization based model (SocialMF) for recommendation in social networks, which incorporate social network trust propagation mechanism [9]; Aiming at modeling the 
particularity of social network systems more accurately and realistically, $\mathrm{Ma} \mathrm{H}$. et al. put forward a recommendation with social trust ensemble (RSTE) approach, which naturally fuses users' tastes and their trusted friends' favors together [7]; Zhou T. C. et al. deemed rating activity and tagging activity play an important role in revealing individuals' opinions and put forward a recommender method by fusing users' tagging information and rating information together [3]. However, most the existing methods cannot performance very well in sparse data condition. The reseach goes into the existing studies and find that multi-dimension data fusion is an efficient way to overcome the challenge in SNR. Therefore, we propose a unified social network recommendation framework that incorporate three famous recommendation models (SoRecUser, SoRecItem and SoRec) and PMF. Our contributions are twofold. First, aim at using aviailable knowledge (tag, rating and trust) jointly in social network applications to make accuate recommendations. Second, based on the properties of PMF, we put forward a unified recommendation framework to slove challeges such data sparity in traditional recommendation systems.

The remainder of the paper article is organized as follows: the basic concepts of the social tag, social trust, PMF and social network recommendation related literals are reviewed in section 2. Detailed idea of the proposed unified social network recommendation framework is explained in Section 3, experiments on public available social network data and the results analysis are conducted in Section 4. Finally, we conclude the paper and present some directions for future work in Section 5.

\section{Literal Review}

\subsection{Social Tag}

The emergence of online social platforms, on the one hand have greatly changed the way we communicate, but on the other hand, they have made more and more crucial information overload problem. Tag technology is deemed as an efficient method to overcome the challenge, because it provides descriptive annotations or keywords to compress information. This enables users to bookmark preferred web pages and network resources (such as pictures, videos, music, blogs, etc.), which are convenient for later information retrieval and fast lookup [10, 11]. Moreover, the usage of tag technology gives birth to a new way for researchers to explore individual's preference and the features of items from social relationships between the users with the help of social tagging systems [1, 12].

Although tags are wildly used, their functions in online systems is rather different: they can usually be defined to associate with any free word relevant to the tagged item which is often not organized into a pre-defined hierarchy of categories or subcategories [13, 14]. Based on users' motivation or semantic of tags, they can be divided into four categories: standard tags, machine tags, action tags and reactive tags [15-17]. 
Standard tags: they are defined from the view point of users' descriptive intend. This kind of tags are most commonly used by many tagging sites at present. They can be applied with a broad range of purposes, and all tags are handled in the same way no matter what intends and extra supports are provided from the tagging sites, except for search and classification [17].

Machine Tags: they are specially designed for machines and structured in machine readable format. The description contains meta information of the resource such as upload date, geographical coordinates, etc. Usually, machine tags are described by a triplet: $<$ namespace, property, value $>$, and users can define them according to the provided syntax [18].

Action Tags: they are machine oriented prescriptive tags, with their operations set by the websites. For instance, action tags available in Delicious offer a "for: User" action tag. Action tags annotate resources as any other tags, so they can be used to organize or locate useful resources. Besides the resource being tagged, additional parameters can be provided using the ":" notation.

Reactive Tags: they are used to annotate user's intentions on the resource being tagged $[16,19]$, for example "toDo", “toRead”, “toDownload”, etc. Some surveys identify this kind of tags as user provided prescriptive tags, however, no way exits to automate these actions. Additionally, these behaviors tend to surpass the tagging sites to impact other device or operations (e.g. the printer, your to-do list, etc.), and might affect other users.

\subsection{Social Trust}

Trust is usually recognized as meaningful social relationships between individuals and of ubiquitous importance to people. A great many researchers have studied it from many disciplines, including computer science, cognitive sciences, sociology, economics, and psychology to explore what is trust, however, they have difficulty in finding an exact definition [20]. From a mental attitude, trust was defined as "a complex attitude of an agent $x$ towards another agent $y$ about the behavior/action relevant for the result (goal) $g$ ” or "the mental counter-part of delegation" [21]. Granovetter M. considered trust as a king of interpersonal different construct procedure [22], while Hosmer L. T. held the opinion that trust was a cognitive process and could be impacted by many factors such as confidence in another's goals or purposes, or the perceived sincerity of another's word [23]. Wei Z. proposed a web of trust notion to represent users' relations and trust relationship of mutual interdependence in social network [24]. Overall, existing definitions of trust generally imply it is a belief that one party is willing to believe or depend on the actions of another party. In addition, four attributes of trust, transitivity, subjective and asymmetric, context dependent and dynamic, are intensively discussed in the existing literatures [25, 26]. 


\subsection{Probability Matrix Factorization}

PMF is deemed as one of the most famous Matrix Factorization models in collaborative filtering $[27,28]$. It defines the conditional distribution over the observed ratings as:

$$
p\left(R \mid U, V, \sigma^{2}\right)=\prod_{i=1}^{N} \prod_{j=1}^{M}\left[\mathcal{N}\left(R_{i j} \mid U_{i}^{T} V_{j}, \sigma^{2}\right)\right]^{I_{i j}}
$$

where $M$ is movie matrix, $N$ is user matrix, $R_{i j}$ is rating matrix with integer rating values, $U_{i}$ and $V_{j}$ are user-specific and movie-specific latent feature vectors respectively; $\mathcal{N}\left(x \mid \mu, \sigma^{2}\right)$ is probability density function of the Gaussian distribution with mean $\mu$ and variance $\sigma^{2} ; I_{i j}$ is the indicator function, with the value equals to 1 if user $i$ rated movie $j$, and otherwise 0. Two zero-mean spherical Gaussian priors can be placed on the following user latent feature vector and movie latent feature vector.

$$
\begin{gathered}
p\left(U \mid \sigma_{U}^{2}\right)=\prod_{i=1}^{N} \mathcal{N}\left(U_{i} \mid 0, \sigma_{U}^{2} \boldsymbol{I}\right) \\
p\left(V \mid \sigma_{V}^{2}\right)=\prod_{j=1}^{M} \mathcal{N}\left(V_{j} \mid 0, \sigma_{V}^{2} \boldsymbol{I}\right)
\end{gathered}
$$

Log function of posterior distribution over the two latent feature vectors can be represented as:

$$
\begin{aligned}
& \operatorname{lnp}\left(U, V \mid R, \sigma^{2}, \sigma_{V}^{2}, \sigma_{U}^{2}\right) \\
& =-\frac{1}{2 \sigma^{2}} \sum_{i=1}^{N} \sum_{j=1}^{M} I_{i j}\left(R_{i j}-U_{i}^{T} V_{j}\right)^{2}-\frac{1}{2 \sigma_{U}^{2}} \sum_{i=1}^{N} U_{i}^{T} U_{i}-\frac{1}{2 \sigma_{V}^{2}} \sum_{j=1}^{N} V_{j}^{T} V_{j} \\
& -\frac{1}{2}\left(\left(\sum_{i=1}^{N} \sum_{j=1}^{M} I_{i j}\right) \ln \sigma^{2}+N D \ln \sigma_{U}^{2}+M D \ln \sigma_{V}^{2}\right) \\
& +P \\
& -4)
\end{aligned}
$$

where $P$ is a constant. Maximized form of formula (2-4) with hyper-parameters fixed is equal to the following objective function:

$$
\frac{1}{2} \sum_{i=1}^{N} \sum_{j=1}^{M} I_{i j}\left(R_{i j}-U_{i}^{T} V_{j}\right)^{2}+\frac{\lambda_{U}}{2} \sum_{i=1}^{N}\left\|U_{i}\right\|_{F}^{2}+\frac{\lambda_{V}}{2} \sum_{j=1}^{M}\left\|V_{j}\right\|_{F}^{2}
$$

where $\lambda_{U}=\frac{\sigma^{2}}{\sigma_{U}^{2}}, \lambda_{V}=\frac{\sigma^{2}}{\sigma_{V}^{2}}$ and $\|\cdot\|_{F}^{2}$ represents the Frobenius norm. 


\subsection{Social Network Recommendation}

SNR is considered as one of the most promising research directions in recommendation system that targets at the social media domain. It focuses on dealing with information overload challenge by combining many new types of data such as tags and explicit online relationships that is related to social network particularity with the existing traditional recommender approaches [29]. Social network has obvious advantages in describing the relation between many information agents, like individuals, groups and other computers. Therefore, SNR is expected to overcome many difficulties, for example data sparsity and cold start exist in traditional recommender systems.

According to the formalized definition of traditional recommendation systems, SNR can be defined as [30]

$$
\forall u_{x} \in U, I^{*}=\arg \max _{i \in I}\left(\mu_{i}+a \mu\left(u_{x}, i\right)+\beta \frac{1}{|Y|} \sum_{y}^{Y} \mu\left(u_{y}, i\right)\right)
$$

where $I^{*}$ is the recommended items for the target user according to ratings, maximum utility and individual's preference; $U$ is user set, $I$ is item set, $i \in I, M=|U|, N=|I|$ and $G=\left(g_{i j}\right)_{M \times N}$ denotes relationship matrix of all users; the mapping $\mu: U \times I \rightarrow R$ is an evaluation function for the recommended results; $Y=\left\{u_{y} \mid u_{y} \in U, x \neq y, g_{x, y} \neq\right.$ $\left.0, g_{x, y} \in G\right\}, \forall \alpha, \beta \in[0,1], \alpha+\beta=1$ and $\mu_{i}$ is a bias variable that represents popular reputation degree of item $i$ in all users.

Generally, SNR falls into collaborative filtering, content-based filtering and hybrid recommendation, and most of the existing social network recommendation methods are based on friendship and other social information fused collaborative filtering [31-34]. In recent years, with the widespread use of social network applications, a variety of famous recommendation models such as social graph based recommendation method, matrix factorization based recommendation model and factor decomposition machine model are proposed successively [35].

\section{Unified Social Network Recommendation Framework}

The proposed unified social network recommendation approach is based three existing recommendation models, namely, SoRecUser, SoRecItem and SoRec. Here we explain the general principle of them first and then introduce the unified social network recommendation framework, which incorporates tags, trust between users, ratings and PMF technology. To facilitate our discussions, Tab. 3-1 lists all the terms and notations that employed in the reminder of this paper. 
Table 3-1. Notations throughout this paper

\begin{tabular}{|c|c|}
\hline Notation & Description \\
\hline \hline$M, N, Q$ & Number of users, items and tags respectively \\
\hline$F$ & Dimension of latent feature \\
\hline$U_{M * F}$ & $M^{*} F$ dimension user latent feature vector \\
\hline$V_{N * F}$ & $N^{*} F$ dimension item latent feature vector \\
\hline$Z_{M * F}$ & $M^{*} F$ dimension trust latent feature vector \\
\hline$T_{Q * F}$ & $Q^{*} F$ dimension tag latent feature vector \\
\hline$R_{i j}$ & Rating of $U_{i}$ on item $V_{j}$ \\
\hline$F_{i k}$ & Tag number that $U_{i}$ mark $\mathrm{T}_{\mathrm{k}}$ \\
\hline$G_{j k}$ & Number of tags that $V_{j}$ marked as $\mathrm{T}_{\mathrm{k}}$ \\
\hline$C_{i l}$ & Trust degree between $U_{i}$ and $Z_{l}$ \\
\hline$\widetilde{R_{l j}}$ & Predicted rating of $U_{i}$ on item $V_{j}$ \\
\hline
\end{tabular}

\subsection{Social Tag Based User Model}

With reference to the analysis in [4], conditional probability distribution function of user $U_{i}$ mark tag $T_{k}$ can be estimated as

$$
p\left(F \mid U, T, \sigma_{F}^{2}\right)=\prod_{i=1}^{M} \prod_{k=1}^{Q} \mathbb{N}\left[\left(F_{i k} \mid g\left(U_{i}^{T} T_{k}\right), \sigma_{F}^{2}\right]^{I_{i k}^{F}}\right.
$$

where $I_{i k}^{F}$ is the indicator function. $I_{i k}^{F}$ is set to 1 if $U_{i}$ tag $T_{k}$ for more than once, and 0 otherwise. The probability distribution of $U$ and $T$ can be calculated by the following equations:

$$
\begin{gathered}
p\left(U \mid \sigma_{U}^{2}\right)=\prod_{i=1}^{M} \mathbb{N}\left(U_{i} \mid 0, \sigma_{U}^{2} \boldsymbol{I}\right) \\
p\left(T \mid \sigma_{T}^{2}\right)=\prod_{k=1}^{Q} \mathbb{N}\left(T_{k} \mid 0, \sigma_{T}^{2} \boldsymbol{I}\right)
\end{gathered}
$$

According to the Bayesian reference, posterior distribution of $U$ and $T$ can be calculated according to formula (3-4).

$$
\begin{aligned}
& p\left(U, T \mid F, \sigma_{F}^{2}, \sigma_{U}^{2}, \sigma_{T}^{2}\right) \propto p\left(F \mid U, T, \sigma_{F}^{2}\right) \times p\left(U \mid \sigma_{U}^{2}\right) \times p\left(T \mid \sigma_{T}^{2}\right) \\
& =\prod_{i=1}^{M} \prod_{k=1}^{Q} \mathbb{N}\left[\left(F_{i k} \mid g\left(U_{i}^{T} T_{k}\right), \sigma_{F}^{2}\right]^{I_{i k}^{F}} \times \prod_{i=1}^{M} \mathbb{N}\left(U_{i} \mid 0, \sigma_{U}^{2} \boldsymbol{I}\right)\right. \\
& \times \prod_{k=1}^{Q} \mathbb{N}\left(T_{k} \mid 0, \sigma_{T}^{2} \boldsymbol{I}\right)
\end{aligned}
$$


The probabilistic graph for the social tag based user model (SoRecUser) [4] can be described in Fig. 3-1.

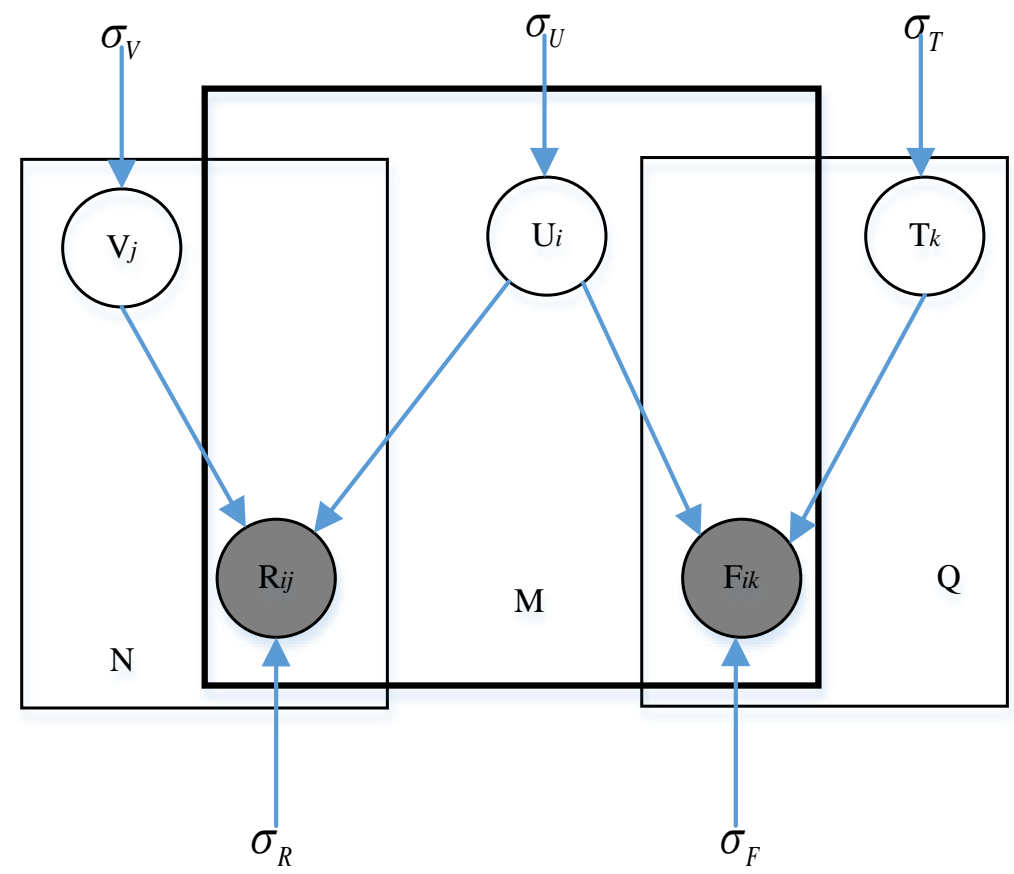

Fig. 3-1. SoRecUser probabilistic graph model

\subsection{Social tag Based Item Model}

Conditional probability distribution function for item $V$ can be calculated as

$$
p\left(G \mid V, T, \sigma_{G}^{2}\right)=\prod_{j=1}^{N} \prod_{k=1}^{Q} \mathbb{N}\left[\left(G_{j k} \mid g\left(V_{j}^{T} T_{k}\right), \sigma_{G}^{2}\right]^{I_{j k}^{G}}\right.
$$

where $I_{j k}^{G}$ is the indicator function, its value is 1 if item $V_{j}$ is tagged by $T_{k}$ for more than one time, and 0 otherwise. The probability distribution of $V$ and $T$ can be calculated by the following equations:

$$
\begin{aligned}
& p\left(V \mid \sigma_{V}^{2}\right)=\prod_{j=1}^{N} \mathbb{N}\left(V_{j} \mid 0, \sigma_{V}^{2} \boldsymbol{I}\right) \\
& p\left(T \mid \sigma_{T}^{2}\right)=\prod_{k=1}^{Q} \mathbb{N}\left(T_{k} \mid 0, \sigma_{T}^{2} \boldsymbol{I}\right)
\end{aligned}
$$

According to the Bayesian reference, posterior distribution of $V$ and $T$ can be represented as 


$$
\begin{aligned}
& p\left(V, T \mid G, \sigma_{G}^{2}, \sigma_{V}^{2}, \sigma_{T}^{2}\right) \propto p\left(G \mid V, T, \sigma_{G}^{2}\right) \times p\left(V \mid \sigma_{V}^{2}\right) \times p\left(T \mid \sigma_{T}^{2}\right) \\
& =\prod_{j=1}^{N} \prod_{k=1}^{Q} \mathbb{N}\left[\left(G_{j k} \mid g\left(V_{j}^{T} T_{k}\right), \sigma_{G}^{2}\right]^{I_{j k}^{G}} \times \prod_{j=1}^{N} \mathbb{N}\left(V_{j} \mid 0, \sigma_{V}^{2} \boldsymbol{I}\right)\right. \\
& \times \prod_{k=1}^{Q} \mathbb{N}\left(T_{k} \mid 0, \sigma_{T}^{2} \boldsymbol{I}\right)
\end{aligned}
$$

And the probabilistic graph for the social tag based item model (SoRecItem) [4] can be described in Fig. 3-2.

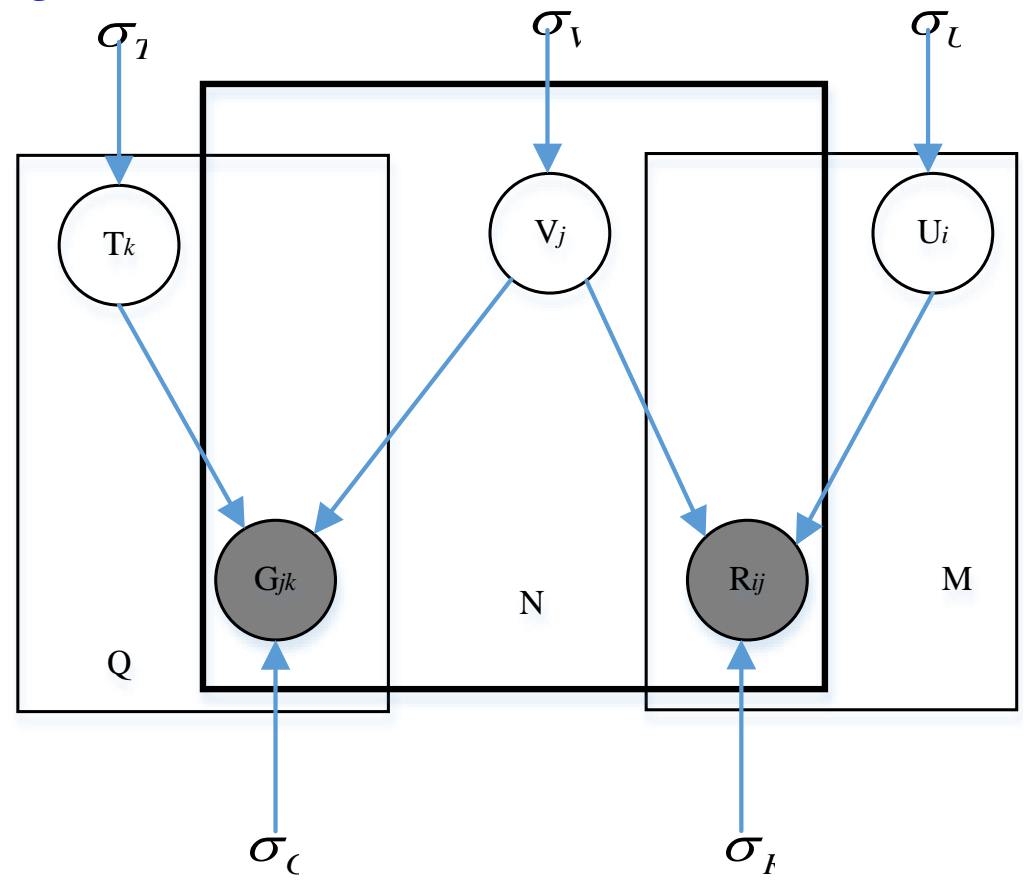

Fig. 3-2. SoRecItem probabilistic graph model

\subsection{Social Trust Based User Model}

Conditional probability distribution function of trust $C_{i l}$ between $U_{i}$ and his (or her) friend $Z_{l}$ can be calculated as

$$
p\left(C \mid U, Z, \sigma_{C}^{2}\right)=\prod_{i=1}^{M} \prod_{l=1}^{M} \mathbb{N}\left[\left(C_{i l} \mid g\left(U_{i}^{T} Z_{l}\right), \sigma_{C}^{2}\right]^{I_{i l}^{C}}\right.
$$

where $I_{i l}^{C}$ is the indicator function, its value is 1 if $U_{i}$ and $Z_{l}$ are friends, and 0 otherwise. The probability distribution of $U$ and $Z$ can be calculated by the following equations.

$$
p\left(U \mid \sigma_{U}^{2}\right)=\prod_{i=1}^{M} \mathbb{N}\left(U_{i} \mid 0, \sigma_{U}^{2} \boldsymbol{I}\right)
$$




$$
p\left(Z \mid \sigma_{Z}^{2}\right)=\prod_{l=1}^{M} \mathbb{N}\left(Z_{l} \mid 0, \sigma_{Z}^{2} \boldsymbol{I}\right)
$$

According to the Bayesian reference, posterior distribution of $U$ and $Z$ can be represented as

$$
\begin{gathered}
p\left(U, Z \mid C, \sigma_{C}^{2}, \sigma_{U}^{2}, \sigma_{Z}^{2}\right)=p\left(C \mid U, Z, \sigma_{C}^{2}\right) \times p\left(U \mid \sigma_{U}^{2}\right) \times p\left(Z \mid \sigma_{Z}^{2}\right) \\
\propto \prod_{i=1}^{M} \prod_{l=1}^{M} \mathbb{N}\left[\left(C_{i l} \mid g\left(U_{i}^{T} Z_{l}\right), \sigma_{C}^{2}\right]^{I_{i l}^{C}}\right.
\end{gathered}
$$

$\times \prod_{i=1}^{M} \mathbb{N}\left(U_{i} \mid 0, \sigma_{U}^{2} \boldsymbol{I}\right) \times \prod_{l=1}^{M} \mathbb{N}\left(Z_{l} \mid 0, \sigma_{Z}^{2} \boldsymbol{I}\right)$

The probabilistic graph for the trust based user model (SoRec) [36] is shown in Fig. 3-3.

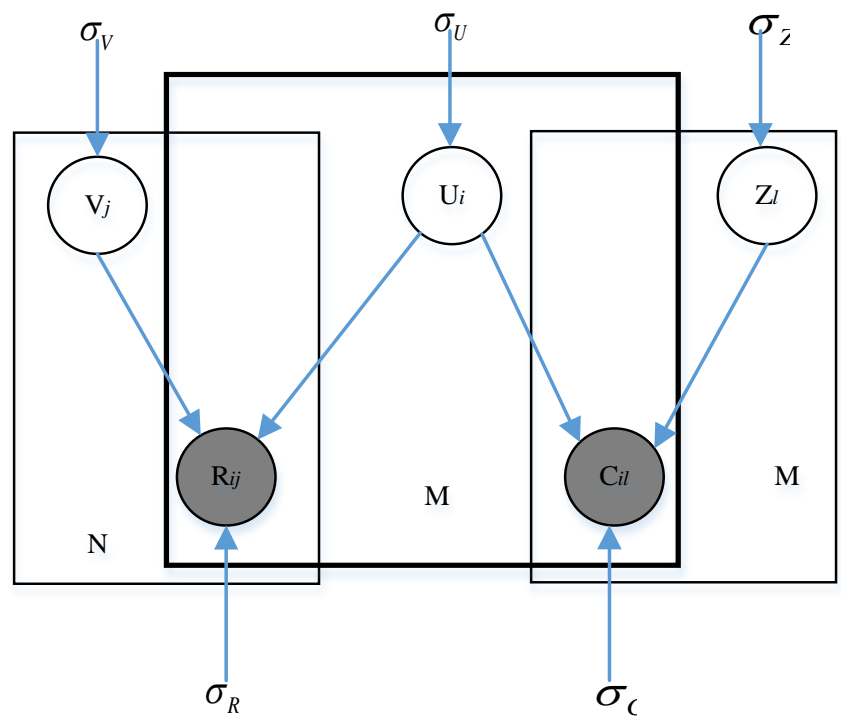

Fig. 3-3. SoRec probabilistic graph model

\subsection{A Fusion Framework with Tag, Trust and Probability Matrix Factorization}

Based on the analysis of probability matrix factorization, social tag based user model, social tag based item model and social trust based user model, we make a fusion of all the approaches and put forward a unified social network recommendation framework, namely, STUIPMF. We get logarithm function of STUIPMF posterior probability distribution function as 


$$
\begin{aligned}
& \ln p\left(U, V, T, Z \mid R, F, G, C, \sigma_{R}^{2}, \sigma_{F}^{2}, \sigma_{G}^{2}, \sigma_{C}^{2}, \sigma_{U}^{2}, \sigma_{V}^{2}, \sigma_{T}^{2}, \sigma_{Z}^{2}\right) \\
= & -\frac{1}{2 \sigma_{R}^{2}} \sum_{i=1}^{M} \sum_{j=1}^{N} I_{i j}^{R}\left(R_{i j}-g\left(U_{i}^{T} V_{j}\right)\right)^{2}-\frac{1}{2 \sigma_{F}^{2}} \sum_{i=1}^{M} \sum_{k=1}^{Q} I_{i k}^{F}\left(F_{i k}-g\left(U_{i}^{T} T_{k}\right)\right)^{2} \\
- & \frac{1}{2 \sigma_{G}^{2}} \sum_{j=1}^{N} \sum_{k=1}^{Q} I_{j k}^{G}\left(G_{j k}-g\left(V_{j}^{T} T_{k}\right)\right)^{2}-\frac{1}{2 \sigma_{C}^{2}} \sum_{i=1}^{M} \sum_{l=1}^{M} I_{i l}^{C}\left(C_{i l}-g\left(U_{i}^{T} Z_{l}\right)\right)^{2} \\
- & \frac{1}{2 \sigma_{U}^{2}} \sum_{i=1}^{M} U_{i}^{T} U_{i}-\frac{1}{2 \sigma_{V}^{2}} \sum_{j=1}^{N} V_{j}^{T} V_{j}-\frac{1}{2 \sigma_{T}^{2}} \sum_{k=1}^{Q} T_{k}^{T} T_{k}-\frac{1}{2 \sigma_{Z}^{2}} \sum_{l=1}^{M} Z_{l}^{T} Z_{l} \\
- & \frac{1}{2}\left(\sum_{i=1}^{M} \sum_{j=1}^{N} I_{i j}^{R}\right) \ln \sigma_{R}^{2}-\frac{1}{2}\left(\sum_{i=1}^{M} \sum_{k=1}^{Q} I_{i k}^{F}\right) \ln \sigma_{F}^{2}-\frac{1}{2}\left(\sum_{j=1}^{N} \sum_{k=1}^{Q} I_{j k}^{G}\right) \ln \sigma_{G}^{2} \\
- & \frac{1}{2}\left(\sum_{i=1}^{M} \sum_{l=1}^{M} I_{i l}^{C}\right) \ln \sigma_{C}^{2}-\frac{1}{2}(M \times F) \ln \sigma_{U}^{2}-\frac{1}{2}(N \times F) \ln \sigma_{V}^{2} \\
- & \frac{1}{2}(M \times F) \ln \sigma_{Z}^{2}-\frac{1}{2}(Q \times F) \ln \sigma_{T}^{2} \quad(3-13) \\
+ & C
\end{aligned}
$$

where $C$ is an independent constant parameter. Maximum equation of (3-13) equals to the following minimization equation:

$$
\mathcal{L}(U, V, T, Z, R, F, G, C)
$$

$$
\begin{aligned}
& =\frac{1}{2} \sum_{i=1}^{M} \sum_{j=1}^{N} I_{i j}^{R}\left(R_{i j}-g\left(U_{i}^{T} V_{j}\right)\right)^{2}+\frac{\lambda_{F}}{2} \sum_{i=1}^{M} \sum_{k=1}^{Q} I_{i k}^{F}\left(F_{i k}-g\left(U_{i}^{T} T_{k}\right)\right)^{2} \\
& +\frac{\lambda_{G}}{2} \sum_{j=1}^{N} \sum_{k=1}^{Q} I_{j k}^{G}\left(G_{j k}-g\left(V_{j}^{T} T_{k}\right)\right)^{2}+\frac{\lambda_{C}}{2} \sum_{i=1}^{M} \sum_{l=1}^{M} I_{i l}^{C}\left(C_{i l}-g\left(U_{i}^{T} Z_{l}\right)\right)^{2} \\
& +\frac{\lambda_{U}}{2} \sum_{i=1}^{M}\|U\|_{F}^{2}+\frac{\lambda_{V}}{2} \sum_{j=1}^{N}\|V\|_{F}^{2}+\frac{\lambda_{T}}{2} \sum_{k=1}^{Q}\|T\|_{F}^{2} \\
& +\frac{\lambda_{Z}}{2} \sum_{l=1}^{M}\|Z\|_{F}^{2}
\end{aligned}
$$

where regularization parameters $\lambda_{F}=\sigma_{R}^{2} / \sigma_{F}^{2}, \lambda_{G}=\sigma_{R}^{2} / \sigma_{G}^{2}, \lambda_{C}=\sigma_{R}^{2} / \sigma_{C}^{2}, \lambda_{U}=\sigma_{R}^{2} / \sigma_{U}^{2}$, $\lambda_{V}=\sigma_{R}^{2} / \sigma_{V}^{2}, \lambda_{T}=\sigma_{R}^{2} / \sigma_{T}^{2}, \lambda_{Z}=\sigma_{R}^{2} / \sigma_{Z}^{2}$, and $\|\cdot\|_{F}$ is the Frobenius norm. According to the stochastic gradient descent method, we get 


$$
\begin{gathered}
\frac{\partial \mathcal{L}}{\partial U_{i}}=\sum_{j=1}^{N} I_{i j}^{R} g^{\prime}\left(U_{i}^{T} V_{j}\right)\left(g\left(U_{i}^{T} V_{j}\right)-R_{i j}\right) V_{j}+\lambda_{F} \sum_{k=1}^{Q} I_{i k}^{F} g^{\prime}\left(U_{i}^{T} T_{k}\right)\left(g\left(U_{i}^{T} T_{k}\right)-F_{i k}\right) T_{k} \\
\quad+\lambda_{C} \sum_{l=1}^{M} I_{i l}^{C} g^{\prime}\left(U_{i}^{T} Z_{l}\right)\left(g\left(U_{i}^{T} Z_{l}\right)-C_{i l}\right) Z_{l}+\lambda_{U} U_{i} \\
\frac{\partial \mathcal{L}}{\partial V_{j}}=\sum_{i=1}^{M} I_{i j}^{R} g^{\prime}\left(U_{i}^{T} V_{j}\right)\left(g\left(U_{i}^{T} V_{j}\right)-R_{i j}\right) U_{i}+\lambda_{G} \sum_{k=1}^{Q} I_{j k}^{G} g^{\prime}\left(V_{j}^{T} T_{k}\right)\left(g\left(V_{j}^{T} T_{k}\right)-G_{j k}\right) T_{k} \\
\quad+\lambda_{V} V_{j} \\
\frac{\partial \mathcal{L}}{\partial T_{k}}=\lambda_{F} \sum_{i=1}^{M} I_{i k}^{F} g^{\prime}\left(U_{i}^{T} T_{k}\right)\left(g\left(U_{i}^{T} T_{k}\right)-F_{i k}\right) U_{i}+\lambda_{G} \sum_{j=1}^{N} I_{j k}^{G} g^{\prime}\left(V_{j}^{T} T_{k}\right)\left(g\left(V_{j}^{T} T_{k}\right)-G_{j k}\right) V_{j} \\
\quad+\lambda_{T} T_{k} \\
\frac{\partial \mathcal{L}}{\partial Z_{l}}=\lambda_{C} \sum_{i=1}^{M} I_{i l}^{C} g^{\prime}\left(U_{i}^{T} Z_{l}\right)\left(g\left(U_{i}^{T} Z_{l}\right)-C_{i l}\right) U_{i}+\lambda_{z} Z_{l}
\end{gathered}
$$

The probabilistic graph for STUIPMF can be described in Fig. 3-4.

To reduce computational complexity, we set regularization parameters $\lambda_{U}=\lambda_{V}=\lambda_{T}=$ $\lambda_{Z}=\lambda_{F}=\lambda_{G}=\lambda_{C}=\lambda$ in the latter experiments.

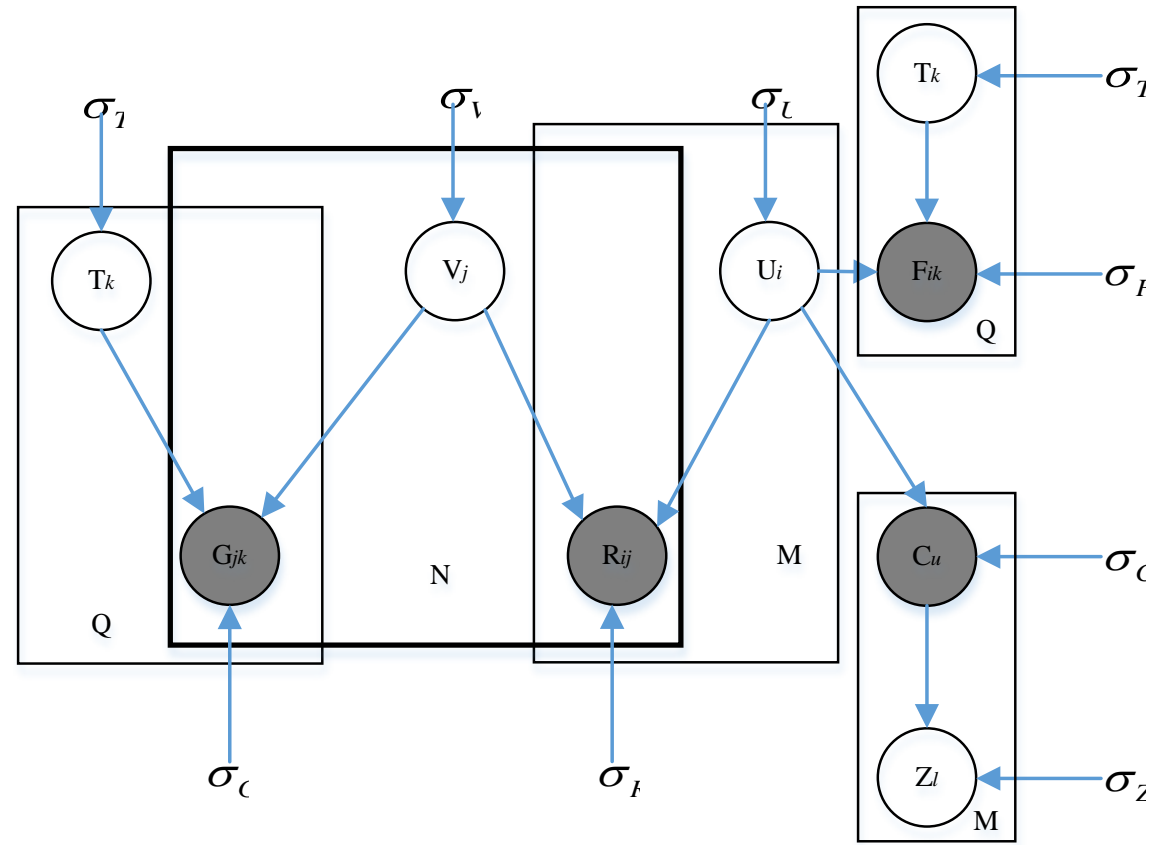

Fig. 3-4. STUIPMF probabilistic graph model 


\subsection{Complexity Analysis}

In the stochastic gradient descent method, the main computation is consumed on objective function $\mathcal{L}$ and the related derivative operations. $R_{i j}, C_{i l}, F_{i k}$ and $G_{j k}$ are all sparse matrixes, so time complexity of $\mathcal{L}$ is $O\left(\rho_{R} f+\rho_{C} f+\rho_{F} f+\rho_{G} f\right)$, where $f$ is the dimension of latent feature vector, $\rho_{R}, \rho_{C}, \rho_{F}$ and $\rho_{G}$ are the number of non-zero items in $R_{i j}, C_{i l}, F_{i k}$ and $G_{j k}$, respectively. Computational complexities of $\frac{\partial \mathcal{L}}{\partial U_{i}}, \frac{\partial \mathcal{L}}{\partial V_{j}}, \frac{\partial \mathcal{L}}{\partial T_{k}}$ and $\frac{\partial \mathcal{L}}{\partial Z_{l}}$ are $O\left(\rho_{R} f+\rho_{C} f+\rho_{F} f\right), O\left(\rho_{R} f+\rho_{G} f\right), O\left(\rho_{C} f\right)$, respectively. Therefore, the overall time complexity of STUIPMF is $\left(\rho_{R} f+\rho_{C} f+\rho_{F} f+\rho_{G} f\right)$ and the computational complexity is linearly to four sparse matrixes $R_{i j}, C_{i l}, F_{i k}$ and $G_{j k}$. All these indicate STUIPMF has good scalability and capable of handling large-scale datasets.

\section{Experiments and Analysis}

In this section, we conduct experiments on publicly available dataset and compare the results with the existing state-of-art recommender methods (PMF, SoRecUser, SoRecItem and SoRec). In the entire process, we address the following three issues:

(1) How does our recommender model compare with the baseline algorithms?

(2) To what extent our recommendation algorithm alleviates the challenge caused by data sparsity?

(3) Whether our recommender approach can handle large-scale datasets efficiently?

\subsection{Dataset}

To test the effectiveness of our recommendation model, we collect a publicly available Last.fm dataset - the hetrec2011-lastfm-2k data ${ }^{1}$, which was released in the 2nd International Workshop on Information Heterogeneity and Fusion in Recommender Systems (HetRec 2011). The dataset was obtained from Last.fm online music system. It contains 1892 users, 17632 items, 11946 tags, 92834 ratings, 12717 friend records, with an average of 98.562 tags per user and 14.891 tags per item. All the users are connected by means of friendship. The relationship between users, tags and music artists in the dataset are structured and stored in [user, tag, artist] tuples.

\subsection{Measures}

In this paper two popular accuracy indicators, the Root Mean Squared Error (RMSE) and the Mean Absolute Error (MAE) [37], are used to evaluate the effectiveness of our recommendation model. RMSE and MAE between the predicted and actual ratings can be obtained according to the following formulas:

\footnotetext{
${ }^{1}$ http://ir.ii.uam.es/hetrec2011/datasets.html
} 


$$
\begin{gathered}
R M S E=\sqrt{\frac{\sum_{(u, i) \in N}\left(R_{u i}-\widehat{R_{u l}}\right)^{2}}{|N|}} \\
M A E=\frac{\sum_{(u, i) \in N}\left|R_{u i}-\widehat{R_{u l}}\right|}{|N|}
\end{gathered}
$$

where $R_{u i}$ are actual ratings for test set $N$ of user-item pairs $(u, i)$, and $\widehat{R_{u \iota}}$ are the predicted value. Usually, $R_{u i}$ can be calculated offline and $\widehat{R_{u \iota}}$ can be calculated from any recommender models.

\subsection{Setup and Metrics}

As listed in Tab. 3-1, M,N,Q, $R_{i j}, F_{i k}, G_{j k}$ and $C_{i l}$ can be acquired from the experimental dataset directly, $U_{M * F}, V_{N * F}, Z_{M * F}$ and $T_{Q * F}$, can be obtained by probabilistic matrix factorization, and $\widehat{R_{l \jmath}}$ can be calculated according to the different social network recommendation models. The calculation of latent feature vectors $\left(U_{M * F}, V_{N * F}\right.$, $Z_{M * F}$ and $\left.T_{Q * F}\right)$ is very important in our experiments, and we take $U_{M * F}$ and $V_{N * F}$ as examples to demonstrate the calculated values with part of Last.fm dataset. Fig. 4-1 shows a user-item matrix with 6 users and 8 rated items. After probabilistic matrix factorization, two latent feature vectors ( $U$ and $V$ ) can be acquired. As show in Fig. 4-2 and Fig. 4-3, $U_{m}$ and $V_{n}$ are the latent feature vectors of users $u_{m}$ and $v_{n}$, respectively.

We randomly split the Last.fm dataset into two non-overlapping sets: a training set and a test set, where the proportion of training data is $80 \%$ and $90 \%$, respectively, and the remainder of the dataset is used as test data. In the experiments, we set training speed parameter $\alpha$ to 0.001 and latent feature vector dimension $F$ to 5 . To find an optimized value for regularization parameter $\lambda$, we set it to different values to examine the final recommendation precision in STUIPMF. As is shown in Fig. 4-4 that there is an obvious influence of $\lambda$ on MAE and RMSE. Specifically, both MAE and RMSE decrease apparently while $\lambda$ increase from 0.004 to 0.007 , and then grow up almost linearly when $\lambda$ exceed 0.007 . Therefore, we set $\lambda$ to 0.007 , which is the mean value of the selected test data in the latter comparison experiments.

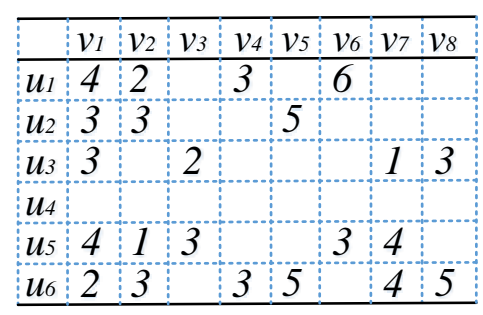

Fig. 4-1. User-item Matrix

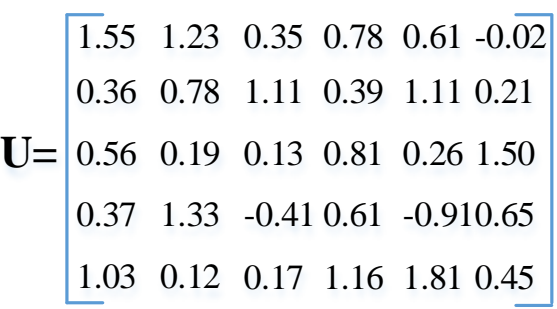

Fig. 4-2. User latent feature vector 


$$
\mathbf{V}=\left[\begin{array}{cccccccc}
1.01 & -0.46 & -0.25 & 0.25 & 1.28 & 0.55 & -0.31 & 0.46 \\
0.17 & -0.88 & -0.73 & 0.03 & 0.67 & 0.21 & -0.64 & 0.78 \\
0.48 & 0.09 & -0.05 & -0.60 & 0.12 & 0.07 & 0.06 & 1.50 \\
-0.38 & 0.69 & -0.61 & -0.29 & 1.99 & 0.44 & 0.81 & 0.65 \\
1.47 & -0.98 & 0.26 & 0.14 & 1.23 & 0.05 & -0.37 & 0.75
\end{array}\right]
$$

Fig. 4-3. Item latent feature vector
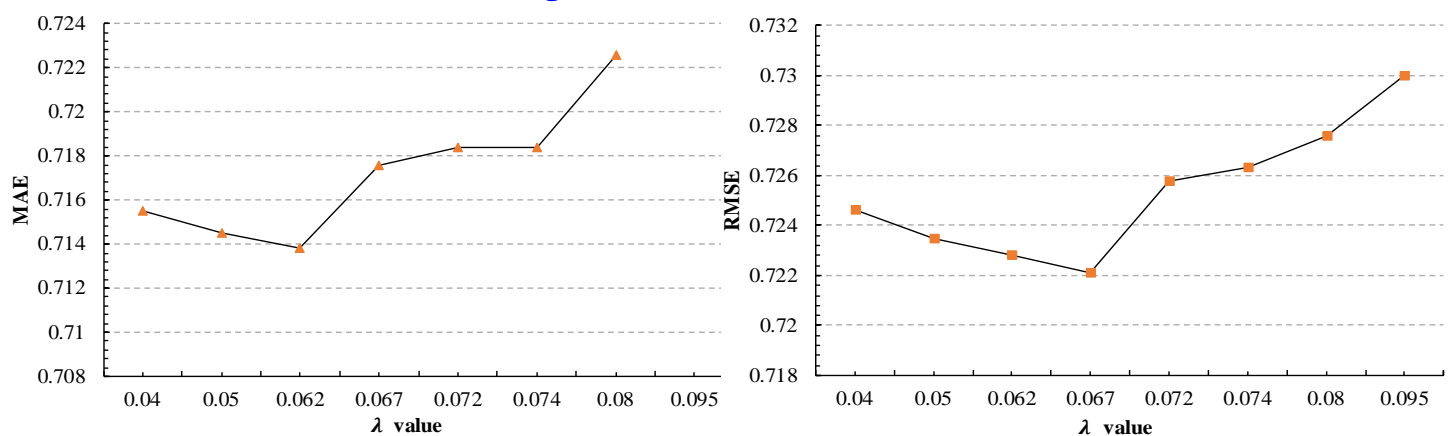

Fig. 4-4. The impact of regularization parameter $\lambda$ on recommendation result in STUIPMF

\subsection{Comparisons}

In the experiments, the compared approaches include:

(1) PMF: it is one of the most well-known models in matrix factorization, and we have explained its basic principle in detail in Section 2.3.

(2) SoRecUser: this is a promising method that takes both items' ratings and users' tags into account. Its objective function is shown in Equation (3-4).

(3) SoRecItem: this method makes full use of both users' ratings and items' tags to generate recommendations based on the probability calculated by Equation (3-8).

(4) SoRec: the method uses both users' ratings and users' trust. Its objective function is shown in Equation (3-12).

(5) STUIPMF: this is the method proposed in the paper. Its objective function is shown in Equation (3-13).

\subsubsection{Performance Comparison}

Tab. 4-1 and Tab. 4-2 report the recommendation results by comparing approaches while latent feature dimension value $F$ is set to 5 and 10 . The results indicate that:

-STUIPMF outperforms PMF, SoRecUser, SoRecItem and SoRec with different latent feature dimension values, no matter take $80 \%$ or $90 \%$ of the dataset as training set. For instance, in performance comparisons using $80 \%$ of the dataset as training set, STUIPMF attains 0.7138 in terms of MAE when latent feature dimension value $F$ is set to 5, while under the same condition, PMF achieves 0.7461 for the counterpart test. 
-Both SoRecUser and SoRecItem integrate social tag information and achieve better performance than PMF; SoRec incorporates social trust information, and get better performance than PMF; Meanwhile, STUIPMF make an integrated usage of social tag, social trust and rating, and outperforms all other methods listed in Tab. 4-1. This implies the importance of fusing multidimensional information in social network recommendation.

-With the increase of latent feature dimension value $F$, the recommendation accuracy tends to decrease for PMF, SoRecUser, SoRecItem, SoRec and STUIPMF.

Table 4-1. Performance comparisons using $80 \%$ of the dataset as training set

\begin{tabular}{|c|c|c|c|c|}
\hline \multirow{2}{*}{ Methods } & \multicolumn{2}{|c|}{$F=5$} & \multicolumn{2}{c|}{$F=10$} \\
\cline { 2 - 5 } & MAE & RMSE & MAE & RMSE \\
\hline PMF & 0.7461 & 0.7526 & 0.7493 & 0.7422 \\
\hline SoRecUser & 0.7258 & 0.7330 & 0.7228 & 0.7269 \\
\hline SoRecItem & 0.7327 & 0.7391 & 0.7309 & 0.7347 \\
\hline SoRec & 0.7350 & 0.7427 & 0.7349 & 0.7355 \\
\hline STUIPMF & 0.7138 & 0.7211 & 0.7124 & 0.7188 \\
\hline
\end{tabular}

Table 4-2. Performance comparisons using $90 \%$ of the dataset as training set

\begin{tabular}{|c|c|c|c|c|}
\hline \multirow{2}{*}{ Methods } & \multicolumn{2}{|c|}{$F=5$} & \multicolumn{2}{c|}{$F=10$} \\
\cline { 2 - 5 } & MAE & RMSE & MAE & RMSE \\
\hline PMF & 0.7441 & 0.7512 & 0.73283 & 0.7398 \\
\hline SoRecUser & 0.7203 & 0.7276 & 0.7188 & 0.7237 \\
\hline SoRecItem & 0.7309 & 0.7380 & 0.7302 & 0.7376 \\
\hline SoRec & 0.7277 & 0.7417 & 0.7335 & 0.7371 \\
\hline STUIPMF & 0.7099 & 0.7175 & 0.7072 & 0.7124 \\
\hline
\end{tabular}

\subsubsection{Data Sparsity}

Data Sparsity is deemed as one of the greatest challenges in recommender systems. To examine the performance of the proposed STUIPMF in different data sparsity conditions, we group all the users in the $80 \%$ training dataset based on users' rating numbers. Here, users are divided into 6 groups: “1-5”, “6-25”, “26-45”, “46-65”, “66-80”, “81-160” and “>160”. Fig. 4-5 gives an overall picture of the distribution on different range of users' rating number. It shows that $50.8 \%$ of the users have less than 5 ratings, and $20.6 \%$ of the users have $6-25$ ratings. 


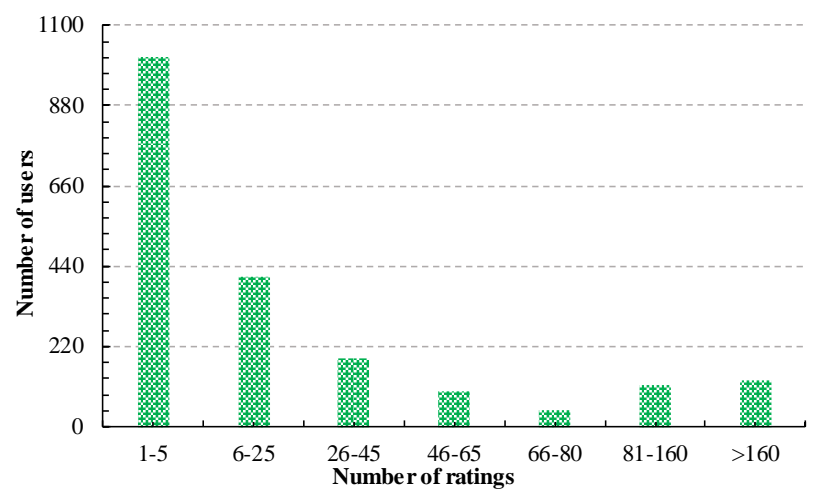

Fig. 4-5. Distribution of user types
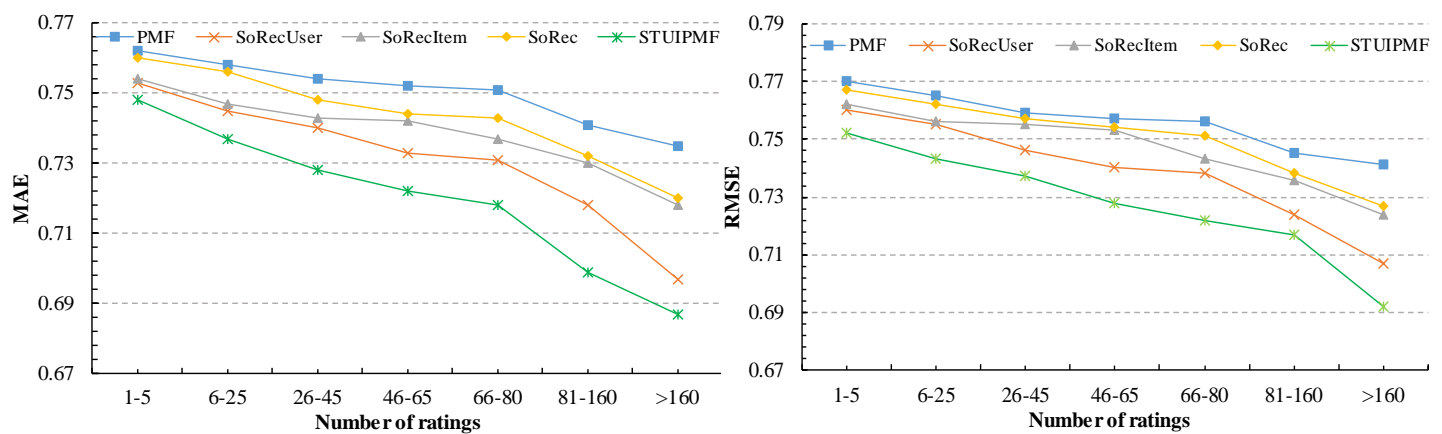

Fig. 4-6. Performance comparison on different user categories

From Fig. 4-6, we can find that the proposed STUIPMF outperforms PMF, SoRecUser, SoRecItem and SoRec on different user types, evaluated by MAE and RMSE. The reason lies in the fact that STUIPMF incorporate three categories of social data sources (tag, rating and trust). Under conditions of sparse data, for example, seldom rating exits, user latent feature vector can be obtained by many ways, such as user-item matrix or user-trust matrix. Therefore, STUIPMF can effectively alleviate data sparsity problem to a certain extent.

\section{Conclusion}

This paper investigates the characteristics of social network applications and the exiting recommender approaches. Based on the basic understanding that rating, tag and trust reflect a user's behavior or item's attribute, the paper put forward a unified recommender model STUIPMF to incorporate three different kind of information together within probability matrix factorization framework. The computational analysis shows that STUIPMF has good scalability and able to handle very large-scale datasets. In addition, performance comparison results indicate the proposed model outperforms PMF, SoRecUser, SoRecItem and SoRec while evaluated by MAE and RMSE.

In the future, we will focus on two worthy directions: (1) how to choose more reasonable 
parameter values to improve STUIPMF and (2) how to incorporate other available information, such friendship and location context into our model to achieve higher recommendation accuracy.

\section{Acknowledgement}

This work is partly supported by the Ministry of Education, the Humanities and Social Sciences Search project (Grant No. 14YJC630210), the Zhijiang Youth Action Project: study on mobile e-commerce recommendation (Grant No. G306), the Zhejiang Public Technology Research and Application project (Grant No. 2015C33065), the Zhejiang Province Education Department General Research project (Grant No. Y201431553), the key project of Ningbo education science planning (Grant No. 2017YZD010), the Natural Science Foundation of Zhejiang (Grant No. LY16G020012), the Major Research Projects of Humanities and Social Sciences in Colleges and Universities of Zhejiang (2014GH015), the Zhejiang Association for Business Economics project (Grant No. 2016SJYB01), the Qinglan program for universities in Jiangsu province, the Modern Port Service Industry and Culture Research Center of the Key Research Base of Philosophy and Social Sciences of Zhejiang Province and the China Scholarship Council.

\section{References}

[1] Millen D R, Feinberg J, "Using social tagging to improve social navigation," in Proc. of the Workshop on the Social Navigation and Community based Adaptation Technologies, June 20-20, 2006. Article (CrossRef Link).

[2] $\mathrm{Wu} \mathrm{L}$, Chen E, Liu Q, Xu L, Bao T, Zhang L, "Leveraging tagging for neighborhood-aware probabilistic matrix factorization," in Proc. of the 21st ACM international conference on Information and knowledge management, pp. 1854-1858, October 29 - November 02, 2012. Article (CrossRef Link).

[3] Zhou T C, Ma H, King I, Lyu M R, "TagRec: Leveraging Tagging Wisdom for Recommendation," in Proc. of the 2009 International Conference on Computational Science and Engineering, pp. 194-199, Augst 29-31, 2009. Article (CrossRef Link).

[4] MA H., "Learning to Recommend (Ph.D thesis)," Hong Kong; The Chinese University of Hong Kong, 2009. Article (CrossRef Link).

[5] Yu Z, Wang C, Bu J, Wang X, Wu Y, Chen C, "Friend recommendation with content spread enhancement in social networks," Information Sciences, vol. 309, pp. 102-118, 2015.

Article (CrossRef Link).

[6] Yom-Tov E, Diaz F, "Out of sight, not out of mind: on the effect of social and physical detachment on information need," in Proc. of the 34th international ACM SIGIR conference on Research and development in Information Retrieval, pp. 385-394, July 24-28, 2011. Article (CrossRef Link). 
[7] Ma H, King I, Lyu M R, "Learning to recommend with social trust ensemble," in Proc. of the 32nd international ACM SIGIR conference on Research and development in information retrieval, pp. 203-210, July 19-23, 2009. Article (CrossRef Link).

[8] Cha M, Mislove A, Gummadi K P, "A measurement-driven analysis of information propagation in the flickr social network," in Proc. of the 18th international conference on World wide web, pp. 721-730, April 20-24, 2009. Article (CrossRef Link).

[9] Jamali M, Ester M, "A matrix factorization technique with trust propagation for recommendation in social networks," in Proc. of the fourth ACM conference on Recommender systems, pp. 135-142, September 26-30, 2010. Article (CrossRef Link).

[10] Zlatić V, Ghoshal G, Caldarelli G, "Hypergraph topological quantities for tagged social networks," Physical Review E, vol. 80, no. 3, pp. 1-8, 2009. Article (CrossRef Link).

[11] Hung C-C, Huang Y-C, Hsu J Y-j, Wu D K-C, "Tag-based user profiling for social media recommendation," in Proc. of the Workshop on Intelligent Techniques for Web Personalization \& Recommender Systems at AAAI, pp. 49-55, July 13-14, 2008. Article (CrossRef Link).

[12] Tibély G, Pollner P, Vicsek T, Palla G, "Extracting tag hierarchies," PloS one, vol. 8, no. 12, pp. e84133, 2013. Article (CrossRef Link).

[13] Symeonidis P, Nanopoulos A, Manolopoulos Y, "A unified framework for providing recommendations in social tagging systems based on ternary semantic analysis," IEEE Transactions on Knowledge and Data Engineering, vol. 22, no. 2, pp. 179-192, 2010.

Article (CrossRef Link).

[14] Spyns P, De Moor A, Vandenbussche J, Meersman R, "From folksologies to ontologies: How the twain meet," in Proc. of the OTM Confederated International Conferences on the Move to Meaningful Internet Systems, pp. 738-755, October 29-November 3, 2006.

Article (CrossRef Link).

[15] Iturrioz J, Díaz O, Azpeitia I, "Reactive tags: Associating behaviour to prescriptive tags," in Proc. of the 22nd ACM conference on Hypertext and hypermedia, pp. 191-200, June 06-09, 2011. Article (CrossRef Link).

[16] Ames M, Naaman M, "Why we tag: motivations for annotation in mobile and online media," in Proc. of the SIGCHI conference on Human factors in computing systems, pp. 971-980, April 28 May 3, 2007. Article (CrossRef Link).

[17] Golder S A, Huberman B A, "Usage patterns of collaborative tagging systems," Journal of information science, vol. 32, no. 2, pp. 198-208, 2006. Article (CrossRef Link).

[18] Heymann P, Ramage D, Garcia-Molina H, "Social tag prediction," in Proc. of the 31st annual international ACM SIGIR conference on Research and development in information retrieval, pp. 531-538, July 20-24, 2008. Article (CrossRef Link).

[19] Chi C, Zhou M X, Yang M, Xiao W, Yu Y, Sun X, "Dandelion: Supporting coordinated, collaborative authoring in Wikis," in Proc. of the SIGCHI Conference on Human Factors in Computing Systems, pp. 1199-1202, April 10-15, 2010. Article (CrossRef Link). 
[20] Zhong H, Zhang S, Wang Y, Shu Y, "Study on Directed Trust Graph Based Recommendation for E-commerce System," INTERNATIONAL JOURNAL OF COMPUTERS COMMUNICATIONS \& CONTROL, vol. 9, no. 4, pp. 510-523, 2014. Article (CrossRef Link).

[21] Falcone R, Castelfranchi C, "Social trust: A cognitive approach," Trust and deception in virtual societies; Springer, pp. 55-90, 2001. Article (CrossRef Link).

[22] Granovetter M, "Economic action and social structure: The problem of embeddedness," American journal of sociology, vol. 91, no. 3, pp. 481-510, 1985. Article (CrossRef Link).

[23] Hosmer L T, "Trust: The connecting link between organizational theory and philosophical ethics," Academy of Management Review, vol. 20, no. 2, pp. 379-403, 1995. Article (CrossRef Link).

[24] Wei Z, "A Novel Trust Model Based on Recommendation for E-commere," in Proc. of the 2007 International Conference On Service Systems and Service Management, pp. 1-4, June 09-11, 2007. Article (CrossRef Link).

[25] Ries S, Kangasharju J, Mühlhäuser M, "A classification of trust systems," in Proc. of the OTM Confederated International Conferences on the Move to Meaningful Internet Systems, pp. 894-903, October 29 - November 3, 2006. Article (CrossRef Link).

[26] Yuan W, Shu L, Chao H C, Guan D, Lee Y K, Lee S, "ITARS: trust-aware recommender system using implicit trust networks," IET Communications, vol. 4, no. 14, pp. 1709-1721, 2010. Article (CrossRef Link).

[27] Salakhutdinov R, Mnih A, "Probabilistic Matrix Factorization," Advances in neural information processing systems, vol. 20, no. 1, pp. 1257-1264, 2008. Article (CrossRef Link).

[28] Dueck D, Frey B, Dueck D, Frey B J, Probabilistic sparse matrix factorization: University of Toronto Report, 2004. Article (CrossRef Link).

[29] Wang Z, Sun L, Zhu W, Yang S, Li H, Wu D, "Joint social and content recommendation for user-generated videos in online social network," IEEE Transactions on Multimedia, vol. 15, no. 3, pp. 698-709, 2013. Article (CrossRef Link).

[30] Meng XW, Liu SD, Zhang YJ, Hu X, "Research on social recommender systems (in Chinese)," Ruan Jian Xue Bao/Journal of Software, vol. 26, no. 6, pp. 1356-1372, 2015. Article (CrossRef Link).

[31] Yu L, Pan R, Li Z, "Adaptive social similarities for recommender systems," in Proc. of the fifth ACM conference on Recommender systems, pp. 257-260, October 23-27, 2011. Article (CrossRef Link).

[32] Liu F, Lee $\mathrm{H}$ J, "Use of social network information to enhance collaborative filtering performance," Expert Systems with Applications, vol. 37, no. 7, pp. 4772-4778, 2010. Article (CrossRef Link).

[33] Tang J, Hu X, Liu H, "Social recommendation: a review," Social Network Analysis and Mining, vol. 3, no. 4, pp. 1113-1133, 2013. Article (CrossRef Link).

[34] Yang X, Guo Y, Liu Y, Steck H, "A survey of collaborative filtering based social recommender systems," Computer Communications, vol. 41, pp. 1-10, 2014. Article (CrossRef Link). 
[35] Ulusoy Ö, Tansel A U, Arkun E, "Recommendation and Search in Social Networks," Springer, 2015. Article (CrossRef Link).

[36] Ma H, Yang H, Lyu M R, King I, "SoRec: social recommendation using probabilistic matrix factorization," in Proc. of the 17th ACM conference on Information and knowledge management, pp. 931-940, October 26-30, 2008. Article (CrossRef Link).

[37]Gunawardana A, Shani G, "Evaluating recommender systems," Recommender Systems Handbook; Springer, pp. 265-308, 2015. Article (CrossRef Link).

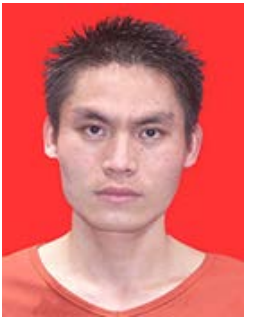

Zhigang Liu received his master's degree in computer science and technology in 2004. Now he is a lecturer in School of Electrical and Mechanical Technology and Wuxi Institute of Technology. At present, his research interests are in the areas of electromechanical integration technology, computer detection and control, and robot technology.

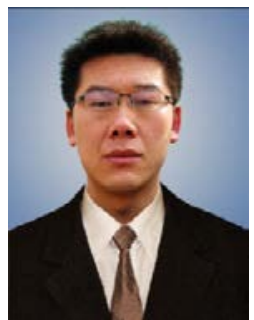

Haidong Zhong is an associate professor in the faculty of Logistics and E-commerce School in Zhejiang Wanli University. He received his B.S in computer science and technology in 2004 and his $\mathrm{PhD}$ in cartography and geography information system from East China Normal University in 2011. At present, his main research interests are in mobile e-commerce, personalized recommendation and business intelligence. 